\title{
Guest editorial: Institute of Hematology and Blood Diseases Hospital in China
}

\author{
Hideo $\operatorname{Ema}^{1}$
}

Received: 6 February 2016 / Revised: 25 February 2016 / Accepted: 1 March 2016 / Published online: 10 March 2016

(C) The Japanese Society of Hematology 2016

In recent years, the contributions of Chinese scientists have played indispensable roles in the development of scientific knowledge and development of novel technologies. Research funding in China provided by the central and local governments has kept pace with economic growth, and the number of scientific publications from China has increased significantly in the past decade. Many talented young people are interested not only in practicing medicine, but also in advancing medical science, and many such investigators also wish to remain in China to conduct their own scientific research.

The Institute of Hematology and Blood Diseases Hospital, located in downtown China's third largest city Tianjin, is one place that offers such opportunities. This institution has the largest hematology hospital and collection of laboratories focused on hematology research in China. The hospital itself was established in the 1950s and later merged with two additional organizations, the Chinese Academy of Medical Sciences and Peking Union Medical College. The resulting center is now well-known in China for basic and clinical studies of normal and abnormal hematopoiesis. This issue of International Journal of Hematology includes four review articles outlining recent progress in the study of hematopoietic stem cells (HSCs) written by members of this institute.

Cell cycle and fate decisions are critically important phenomena in HSCs. Most HSCs may remain quiescent, in a state of reduced basal activity that has been likened

Hideo Ema

hema@ihcams.ac.cn

1 Institute of Hematology and Blood Diseases Hospital, Chinese Academy of Medical Sciences and Peking Union Medical College, 288 Nanjing Rd., Tianjin 300020, China to hibernation, for extended periods, but it is not until they begin cycling that they become functional, either by selfrenewal or differentiation into progenitor cells. HSCs are stimulated by extracellular and intracellular signaling, and their activity is regulated through cell cycle and transcriptional mechanisms. Sha Hao and Chen Chen, together with Tao Cheng, the director of the institute, who was the first to study HSC cell cycle regulators, provide an overview of our current understanding of how the HSC cell cycle is governed. Interestingly, these authors propose that Cyclindependent kinase inhibitors function in cell fate determination during the cycle. Zhao Wang and I focus on extracellular signaling pathways that control HSCs. Even after the discoveries of many self-renewal-related molecules, stem cell factor and thrombopoietin remain two essential cytokines that regulate HSC activity. Weiping Yuan and his colleagues review the rapidly expanding field of intracellular signaling through mTOR and discuss different roles played by mTORC 1 and $\mathrm{mTORC} 2$ in HSC regulation.

HSCs cannot be maintained in vitro without cytokines. However, to date it has not been possible to control HSC fate using cytokines because the cell cycle is closely associated with differentiation process in HSCs. Since the discovery of the anti-chronic myeloid leukemia drug imatinib, small compound libraries have permitted new approaches to the discovery of molecularly targeted therapeutics, leading to efforts to expand HSCs ex vivo using the similar strategy. If differentiation is blocked by small molecules, the probability of HSC self-renewal may increase. Yu Zhang and Yingdai Gao provide a review of this important topic, recent developments and associated issues.

Hopefully these review papers will assist students and clinicians alike in gaining a new understanding of and insights into the biology of hematopoietic stem cells. 